\title{
ESTUDIO DE CASO-CONTROL PARA EVALUAR FACTORES DE RIESGO EN LA PRESENTACIÓN DE LEPTOSPIROSIS CANINA EN LA CIUDAD DE LIMA
}

\section{Case-Control Study of Risk Factors for Canine Leptospirosis in Lima}

\author{
Carlos Huerta M. ${ }^{1}$, Vicente Chilón C. ${ }^{1}$, Diego Díaz C. ${ }^{1,2}$
}

\section{Resumen}

El objetivo de este trabajo fue realizar un estudio de caso-control para evaluar factores de riesgo en la presentación de leptospirosis canina en pacientes de la Clínica de Animales Menores de la Facultad de Medicina Veterinaria de la Universidad Nacional Mayor de San Marcos, Lima. Se analizaron datos de las historias clínicas y resultados de análisis serológicos de los años 2002 al 2007. Se trabajó con un grupo caso $(n=54)$ y un grupo control $(n=394)$. Los datos se agruparon en las variables sexo, tamaño, edad, temporada del año y residencia, y se analizaron mediante la prueba de regresión logística. Los factores de riesgo asociados a la enfermedad fueron el sexo (el macho presenta el doble de riesgo que la hembra), el tamaño (los perros grandes presentan el doble de riesgo de presentar la enfermedad versus perros pequeños) y la edad (el riesgo es mayor con la edad).

Palabras clave: leptospirosis, caninos, factores de riesgo, caso-control

\section{Abstract}

The aim of the present study was to describe potential risk factors for canine leptospirosis infections in patients of the Small Animal Clinic at the Veterinary School, San Marcos University, Lima, through a case-control study. Data from clinical records and serological test results from 2002 to 2007 were reviewed. Forty four cases and 394 controls were enrolled into the study. Collected data included sex, size, age, season of the year, and origin, and were analyzed by multivariable logistic regression. Risk factors related to the disease were: sex (male has double risk), size (bigger dogs have two times more risk) and age (risk increased with age).

Key words: leptospirosis, canine, risk factors, case-control

\footnotetext{
${ }^{1}$ Clínica de Animales Menores, Facultad de Medicina Veterinaria, Universidad Nacional Mayor de San Marcos, Lima

${ }^{2}$ E-mail: holadiegoperu@hotmail.com
} 


\section{INTRODUCCIÓN}

La leptospirosis es una enfermedad clínica y zoonótica importante. Aunque presenta una distribución global, algunos serovares presentan una distribución geográfica limitada. Hace dos décadas se le consideraba una enfermedad reemergente y hoy su notificación es obligatoria en muchos países del mundo. Los últimos brotes han permitido que aumente el interés como problema de salud pública, debido a que se han producido tanto formas letales como presentaciones subclínicas poco frecuentes. Es una enfermedad potencialmente mortal, pero tratable (Quinn et al., 2005; Céspedes et al., 2006; Moore et al., 2006; Schaer, 2006). Teniendo esto en consideración, los perros pueden convertirse en centinelas de exposición de este microorganismo en humanos (Moore, 2006); sobre todo en lugares con alta prevalencia de esta enfermedad en humanos, como ocurre en zonas de la selva peruana como Loreto y Madre de Dios (Céspedes, 2005).

Una vez infectado, el animal desarrolla la enfermedad, cuyos signos clínicos se presentarán de acuerdo a variables que incluyen la especie de animal infectado y el serovar de la leptospira infectante. Así, se puede encontrar desde animales que mueren sin presentar signos clínicos (leptospirosis peraguda) a animales que presentan una enfermedad larga (leptospirosis crónica) con recuperación clínica lenta, tornándose portadores por meses, incluso años (Levett, 2001). Estos últimos eliminan leptospira por la orina, contaminando el medio ambiente donde se encuentran, con el consiguiente peligro que significa para otros animales y el hombre.

En una ciudad como Lima, donde la Leptospira encuentra los factores necesarios para su presencia y desarrollo como altos niveles de humedad, es importante conocer los factores de riesgo individuales y medioambientales que tienen los perros para contraer la enfermedad. El conocimiento de los factores locales de riesgo ayudará a adop- tar medidas preventivas contra la enfermedad. Por lo tanto, el objetivo de este trabajo fue realizar un estudio de caso-control para evaluar factores de riesgo en la presentación de leptospirosis canina en una clínica de animales menores en la ciudad de Lima.

\section{Materiales y Métodos}

\section{Tipo de Estudio}

Se desarrolló un estudio retrospectivo de tipo caso-control. El número de muestras de determinó en base a los resultados de Batista et al. (2005), empleando la fórmula de Pértegas y Pita (2002), considerando una seguridad de $95 \%$ y un poder estadístico de $80 \%$.

El grupo caso estuvo constituido por 54 muestras y el grupo control por 394 muestras, correspondientes al periodo 2002-2007. Parte de los datos considerados en el estudio fueron proporcionados por los dueños de las mascotas, y el resto fue recabado de las historias clínicas de la Clínica de Animales Menores y de los registros del Laboratorio de Microbiología de la Facultad de Medicina Veterinaria (FMV), Universidad Nacional Mayor de San Marcos (UNSM), Lima.

\section{Definición de Caso}

Se consideró como Caso a todo can que fue llevado a consulta con signos clínicos de enfermedad renal/hepática aguda, y que al descarte de leptospirosis resultó con títulos mayores o iguales a 1:400 en la prueba de aglutinación microscópica para Leptospira (MAT), prueba recomendada por la Organización Mundial de la Salud (WHO, 2003); y que, además, no haya sido vacunado contra la leptospirosis en los tres meses previos a la presentación de la enfermedad.

En los pocos casos que las muestras dieron como resultado positivo a más de un serovar, se tomó como agente causante al 
que presentó el mayor título, y al haber más de un serovar con el mismo título, se tomó a todos por agentes causantes por asociación de la enfermedad (Ghneim et al., 2007). Los animales con títulos bajos (entre 1:50 y 1:200) no se tomaron en cuenta para el presente trabajo (Ghneim et al., 2007), pese a que clínicamente se les considera positivos e indican necesidad de tratamiento.

Se tomó como signos de enfermedad renal/hepática aguda la letargia, depresión, anorexia, vómito y fiebre alta (sobre $39.5^{\circ} \mathrm{C}$ ), así como el dolor paraespinal lumbar (Madigan et al., 1999; Mc Donough, 2001; Greene, 2003; Schaer, 2006).

\section{Definición de Control}

Como grupo control se consideró a todos los animales que llegaron sanos a la consulta, para revisiones, chequeos o vacunaciones, y a aquellos que llegaron para cirugías estéticas, tratamientos pulguicidas, y baños. Se incluyó, además, a los que presentaron signos de enfermedad renal o hepática aguda, y obtuvieron resultado serológico negativo a la prueba MAT para descarte de leptospirosis.

\section{Análisis de Datos}

Las variables bajo estudio fueron sexo, edad (<1, 1-3, 4-7, > 7 años), tamaño (pequeño, mediano, grande), temporada del año (verano, otoño, invierno, primavera), y lugar de residencia en Lima (centro, norte, sur, este, oeste). En los perros de raza indefinida (criollos y cruzados) se tomó en cuenta el peso para su categorización: pequeños (menores de $10 \mathrm{~kg}$ ), medianos (entre 10 y $20 \mathrm{~kg}$ ) y grandes (mayores de $20 \mathrm{~kg}$ ). En los casos donde el paciente tuvo más de un lugar de residencia se tomó en cuenta aquel donde pasó el mayor tiempo.

Se utilizó la prueba de regresión logística para determinar los Odds Ratio (OR) ajustados para los factores de riesgo y un nivel de significancia de 0.05 (Spiegel, 1991; Daniel, 1996; Velásquez y Rey, 2007).

\section{Resultados}

En el grupo caso se observaron las siguientes tendencias: el $68.5 \%$ (37/54) fue macho, el $48.1 \%$ de tamaño grande, el $25.9 \%$ mayor de 7 años, el $47.2 \%$ estuvo localizado en la zona de Lima Centro, y el $37 \%$ se presentó a la evaluación clínica en la primavera. En el grupo control se observó que el $57.4 \%$ (226/394) de los animales fue macho, el $43.1 \%$ fue de tamaño pequeño, el $49.5 \%$ menor de un año de edad, el $57.4 \%$ fue residente de la zona de Lima Centro, y el 38.6\% llegó a la clínica en la temporada de primavera.

El serovar con mayor frecuencia fue canicola $(50.0 \%)$, seguido por el serovar icterohaemorragiae (29.6\%) (Cuadro 1). Casos de asociación ocurrieron entre los serovares canicola e icterohaemorragiae en 2 casos $(3.7 \%)$.

En la evaluación multivariada realizada a los grupos caso y control se hallaron asociaciones significativas en la variable sexo, tamaño y edad. En la variable sexo, la característica hembra mostró ser un factor de protección con relación a la característica macho. En el tamaño se observó que los perros de tamaño grande mostraron casi dos veces mayor riesgo de presentar la enfermedad en comparación a los perros de raza pequeña $(\mathrm{OR}=1.9, \mathrm{p}<0.05)$. En la variable edad, todos los conjuntos etarios mayores a un año mostraron mayor riesgo que los menores a un año (Cuadro 2).

Se hallaron diferencias significativas entre los factores de riesgo en la presentación de leptospirosis canina (variables sexo, tamaño y edad). En la variable sexo, se observó que la característica hembra es un factor de protección para los perros, mostrando casi la mitad del riesgo de presentar la enfermedad con relación al macho $(\mathrm{OR}=0.6$, $\mathrm{p}<0.05)$. Similares resultados se encontraron en EEUU y Canadá entre 1970 a 1998 (OR= $1.6, \mathrm{p}<0.05)$ y en EEUU entre 1997 y 2002 $(\mathrm{OR}=3.2$. $\mathrm{p}<0.05)$ (Ward et al., 2002, 2004a). 
Cuadro 1. Canes que resultaron positivos a serovares de Leptospira, mediante la pruebas de microaglutinación (periodo 2002-2007) ${ }^{1}$

\begin{tabular}{lcccccc}
\hline \multicolumn{5}{c}{ Serovares } & & Total \\
\cline { 2 - 6 } & canicola & icterohaemorragiae & georgia & grippothypposa & can-ict $^{2}$ & \\
\hline Total & 27 & 16 & 8 & 1 & 2 & 54 \\
Porcentaje & 50.0 & 29.6 & 14.8 & 1.9 & 3.7 & 100.0 \\
\hline 1 & Serovares evaluados por el Laboratorio de Microbiología y & Parasitología, Facultad de Medicina \\
Veterinaria, Universidad Nacional Mayor de San Marcos, Lima & & & \\
canicola e icterohaemorragiae presentan el mismo título & &
\end{tabular}

Cuadro 2. Resultados del Análisis de Odds Ratio ajustado entre los grupos Caso y Control para evaluar factores de riesgo en la presentación de leptospirosis canina

\begin{tabular}{llcc}
\hline Variable & & OR ajustado $^{1}$ & IC 95\% $^{2}$ \\
\hline Sexo & Macho & 1 & \\
\multirow{2}{*}{ Tamaño } & Hembra & 0.6 & $(0.3-0.9)^{\mathrm{a}}$ \\
& Pequeño & 1 & \\
& Mediano & 1.0 & $(0.5-2.1)$ \\
Gdad & Grande & 1.9 & $(1.1-3.3)^{\mathrm{b}}$ \\
& Menor 1 año & 1 & \\
& De 1 a 3 años & 5.6 & $(2.7-11.6)^{\mathrm{b}}$ \\
& De 3 a 5 años & 7.4 & $(3.3-16.8)^{\mathrm{b}}$ \\
& De 5 a 8 años & 19.2 & $(9.1-40.4)^{\mathrm{b}}$ \\
Temporada del año & Mayor 8 años & 9.3 & $(4.5-19.4)^{\mathrm{b}}$ \\
& Verano & 1 & \\
& Otoño & 0.5 & $(0.2-1.1)$ \\
& Invierno & 0.8 & $(0.4-1.7)$ \\
& Primavera & 0.6 & $(0.3-1.1)$ \\
Residencia & Norte & 1 & \\
& Sur & 0.4 & $(0.5-3.5)$ \\
& Centro & 0.2 & $(0.2-1.4)$ \\
& Este & 0.2 & $(0.3-1.8)$ \\
\hline
\end{tabular}

${ }_{1}^{1}$ Odds ratio ajustado (análisis multivariado - regresión logística)

${ }^{2}$ Intervalo de confianza

a Factor de protección en análisis multivariado $(p<0.05)$

${ }^{b}$ Factor de riesgo en análisis multivariado $(p<0.05)$ 
En la variable tamaño, los perros de raza grande mostraron casi el doble de riesgo de presentar la enfermedad comparado con los de raza pequeña $(\mathrm{OR}=1.9, \mathrm{p}<0.05)$. No se encontró datos comparativos, dado que otros autores categorizaron esta variable de distinta manera, mayormente por razas o grupos raciales.

El riesgo de presentar leptospirosis estuvo asociado con los grupos etarios, especialmente en los grupos 5 a 8 años y mayores de 8 años (10 y 8 veces más riesgo, respectivamente). Batista et al. (2005) en Brasil reportaron el doble de riesgo en perros mayores de un año comparado con perros de menor edad $(\mathrm{OR}=2.68, \mathrm{p}<0.05)$. Ward et al. $(2002,2004)$ también reportan mayores niveles de riesgo en perros de 4 a 7 años en comparación con perros menores de un año. La mayor posibilidad de que los perros adultos se infecten con esta bacteria está dada por el mayor contacto con la calle que tienen en relación a perros jóvenes, que por lo general no salen de su casa. Si a esto se le agrega factores de riesgo, tales como raza grande y sexo macho, se tiene la descripción perfecta de los animales que comúnmente se ve en las calles; sean perros sin dueño o con dueño, canes que se encuentran en las calles la mayor parte del día, sea por costumbre del animal o por descuido del propietario. En comparación, los perros de raza pequeña y mediana, así como las hembras son los que permanecen más tiempo en casa, son perros falderos que, usualmente, se cuidan con más esmero.

Un caso diferente es el reportado por Ghneim et al. (2007), quienes encuentran 16 veces mayor riesgo de presentación de la enfermedad en perros menores a un año con relación a perros de entre 1 a 3 años de edad $(\mathrm{OR}=16.50, \mathrm{p}<0.05)$; situación que se puede observar en lugares donde se confinan a las mascotas en criaderos, donde la trasmisión ocurre de madre/padre a cría, y donde el serovar involucrado es el canicola.

La ausencia de diferencia significativa entre las temporadas del año puede ser resultado de la alta humedad que hay en Lima durante todo el año, además de la permanente presencia de roedores y animales callejeros en casi toda la capital. Al mismo tiempo, se suma la deficiente recolección y eliminación de basura, la falta de drenaje de aguas empozadas y el riego por inundación con aguas sin tratar de parques y bermas centrales, presencia de canales y su poco cuidado, factores que pueden ser determinantes para la infección y el desarrollo de la enfermedad.

La escasa información, muchas veces, difícil de corroborar, impidió analizar otras variables externas a las mascotas, pero que son de importancia, como charcos de agua cerca de la vivienda, presencia de basura en la calle, convivencia con otras especies animales, presencia de roedores, salida a la calle con correa o con dueño, perros callejeros y la raza de las mascotas (Ward, 2002; Vieira et al., 2003; Ward et al., 2004b).

\section{Conclusiones}

El sexo, el tamaño y la edad del perro son factores de riesgo en la presentación de leptospirosis en canes que fueron pacientes de la Clínica de Animales Menores de la Facultad de Medicina Veterinaria, Universidad Nacional Mayor de San Marcos.

\section{Agradecimientos}

Los autores expresan su agradecimiento el Laboratorio de Microbiología de la Facultad de Medicina Veterinaria de la Universidad Nacional Mayor de San Marcos por el acceso a la información de los resultados de laboratorio. 


\section{Literatura Citada}

1. Adler B, Chappel RJ, Faine S. 1982. The sensitivities of different immunoassays for detecting leptospiral antigen. Zentralbl Bakteriol Mikrobiol Hyg [A]. 252(3): 405-413.

2. Batista C, Alves C, Azevedo $S$, Vasconcellos $S$, Morais Z, Clementino I, Alves F, Lima F, Araújo Neto J. 2005. Soroprevalência e fatores de risco para a leptospirose em cães de Campina Grande, Paraíba. Arq Bras Med Vet Zootec 57: 179-185.

3. Céspedes M. 2005. Leptospirosis: Enfermedad zoonótica reemergente. Rev Peru Med Exp Salud Pública 22: 290-301.

4. Céspedes M, Balda L, Gonzáles D, Tapia R. 2006. Situación de la leptospirosis en el Perú 1994-2004. Rev Peru Med Exp Salud Pública 23: 56-65.

5. Daniel W. 1996. Bioestadística. Base para el análisis de las ciencias de la salud. México: Ed Limusa. 878 p.

6. Ghneim G, Viers J, Chomel B, Kass P, Descollonges D, Johnson M. 2007. Use a case-control study and geographic information system to determine environmental and demographic risk factors for canine leptospirosis. Vet Res 38: 37-50.

7. Greene C. 2003. Canine leptospirosis a re-emerging disease. In: World Congress Proceedings of the World Small Animal Veterinary Association. Bangkok, Thailand.

8. Levett P. 2001. Leptospirosis. Clin Microbiol Rev 14:296-326.

9. Madigan M, Martinko J, Parker J. 1999. Brock, Biología de los microorganismos. $8^{\text {va }}$ ed. Madrid: Prentice Hall Iberia. $200 \mathrm{p}$.

10. Mc Donough P. 2001. Leptospirosis en caninos - estado actual. En: Carmichael L. Recent advances in canine infectious diseases. [Internet]. Disponible en: http:// www.ivis.org/advances/infect_dis_carmichael/ mcdonough_es/ivis.pdf
11. Moore G, Guptill L, Glickman N, Caldanaro R, Aucoin D, Glickman L. 2006. Canine leptospirosis, United States, 2002-2004. Emerg Infect Dis 12(3): 501-503.

12. Pértegas S, Pita S. 2002. Cálculo del tamaño muestral en estudios de casos y controles. En: Metodología de la investigación. Cad Aten Primaria. 9: 148-150. Unidad de Epidemiología Clínica y Bioestadística. Complexo Hospitalario Juan Canalejo. La Coruña, España. [Internet] [19 Agosto 2007] Disponible: http://www.fisterra.com/mbe/investiga/ muestra _casos/muestra_casos2.pdf

13. Quinn P, Markey B, Carter M, Donnelly W, Leonard F. 2005. Microbiología y enfermedades infecciosas veterinarias. Zaragoza: Ed Acribia. 678 p.

14. Schaer M. 2006. Medicina clínica del perro y el gato. Barcelona: Ed Masson. $576 \mathrm{p}$.

15. Spiegel M. 1991. Estadística. $2^{\text {da }}$ ed. México: Mc Graw Hill-Interamericana. $556 \mathrm{p}$.

16. Velásquez A, Rey N. 2007. Metodología de la investigación científica. Lima: Ed San Marcos. 311 p.

17. Vieira A, Botazzo Á, Claret de Oliveira R, Gibson F, Eckehardt E, Lemos R, de Freitas J. 2003. Fatores de risco associados à leptospirose em cães do município de Londrina - PR. Ciências Agrárias 24: 27-34.

18. Ward M. 2002. Seasonality of canine leptospirosis in the United States and Canada and its association with rainfall. Prev Vet Med 56: 203-213.

19. Ward M, Glickman L, Guptill L. 2002. Prevalence and risk factors for leptospirosis among dogs in the United States and Canada: 677 cases (19701998). JAVMA220: 53-58.

20. Ward M, Guptill L, Prahl A, Wu C. 2004a. Serovar-specific prevalence and risk factors for leptospirosis among dogs: 90 cases (1997-2002). JAVMA 224: 1958-1963. 
21. Ward M, Guptill L, Wu C. $2004 b$. Evaluation of environmental risk factors for leptospirosis in dogs: 36 cases (19972002). JAVMA 225: 72-77.

22. [WHO] World Health Organization. 2003. Human leptospirosis: guidance for diagnosis, surveillance and control. WHO Library Cataloguing-in-Publication Data. [Internet] [01 April 2008]. Available in: http://whqlibdoc.who.int/hq/2003/ WHO_CDS_CSR_EPH_2002.23.pdf 